\title{
AUTONOMOUS DETECTION OF VEHICULAR WHEEL ALIGNMENT PARAMETERS
}

\author{
${ }^{1}$ Aaron Ameerali ${ }^{*}$, Nadine Sangster ${ }^{2}$ and Gerard Ragbir ${ }^{3}$ \\ ${ }^{1,2,3}$ Department of Manufacturing \& Design Engineering, The University of the \\ Trinidad \& Tobago \\ ${ }^{1}$ Email: aaron.ameerali@utt.edu.tt $*($ Corresponding author) \\ ${ }^{2}$ Email: nadine.sangster@utt.edu.tt \\ ${ }^{3}$ Email: gerard.ragbir@outlook.com
}

\begin{abstract}
Vehicular technology has improved tremendously in the last few decades. Drivers and passengers are now being made more aware of their surroundings as well as the state of their cars, ergo becoming increasingly capable of making better decisions. These 'smart-vehicles' are directed by microcontrollers and microprocessors where a network of sensors and actuators provide contextual feedback for the user. Some of these features include parking and reverse assistance, collision avoidance and cruise control. In the coming years, this trend will undergo unprecedented growth as the technologies become cheaper to manufacture and implement. In fact, more advanced systems now alert the driver to realtime critical failures and problematic conditions while the simpler ones do so upon start-up. This paper provides a tested framework for a potential sensing system to alert the driver when the vehicle alignment is off.
\end{abstract}

Vehicle misalignment can become an issue quickly as the following can result:

1. Increased tire tread wear leading to reduced traction with the road's surface and ultimately higher chances of accidents as well as more frequent replacement of the tires becoming necessary.

2. Uneven friction at contact between the road and tire can increase the resistance resulting in higher fuel consumption by the engine.

3. Strain on multiple components within the braking system and suspension as misalignment can cause drift while in motion and additionally uneven braking. A damaged suspension is quite expensive to repair or replace.

Early detection of the extent of misalignment can lead to decreased expenditure in the areas of maintenance and fuel consumption, contributing to an increase in reliability. Since many drivers, however experienced they are, may at times be ignorant of the degree of misalignment their vehicle possesses, adding this technology can serve as a potential remedy ultimately improving the user experience and vehicle longevity.

Keywords: Mesh sensor network, Smart detection, Vehicle alignment.

https://doi.org/10.47412/BOQW8777

\section{Introduction}

Wheel Alignment for automotive vehicles arose from the desire to maintain maximum traction and stability between the road and tires. Despite the default desired state which manufacturers usually implement before a vehicle rolls out of the assembly line, the wheels usually become susceptible to deviations about their various Cartesian axes, i.e. $\mathrm{x}, \mathrm{y}$ and $\mathrm{z}$. These deviations across axes can vary in occurrence due to the 
implemented suspension type; suspension types include the McPherson Strut, Double-Wishbone (also known as the Upper-and-Lower A arm) and Multilink Suspension to name a few which are referred to as independent suspensions and vary from dependent and semi-independent types as they usually support each wheel without affecting the remaining wheels and supports [1]. The deviations have been limited to three key parameters which are named: Camber, Caster (or Castor) and Toe as outlined universally.

Camber refers to the angle the wheel forms from the vertical axis through the middle of the wheel (when viewed looking from the front or rear) relative to the road [2]. Figure 1 below shows what is referred to as positive and negative camber.

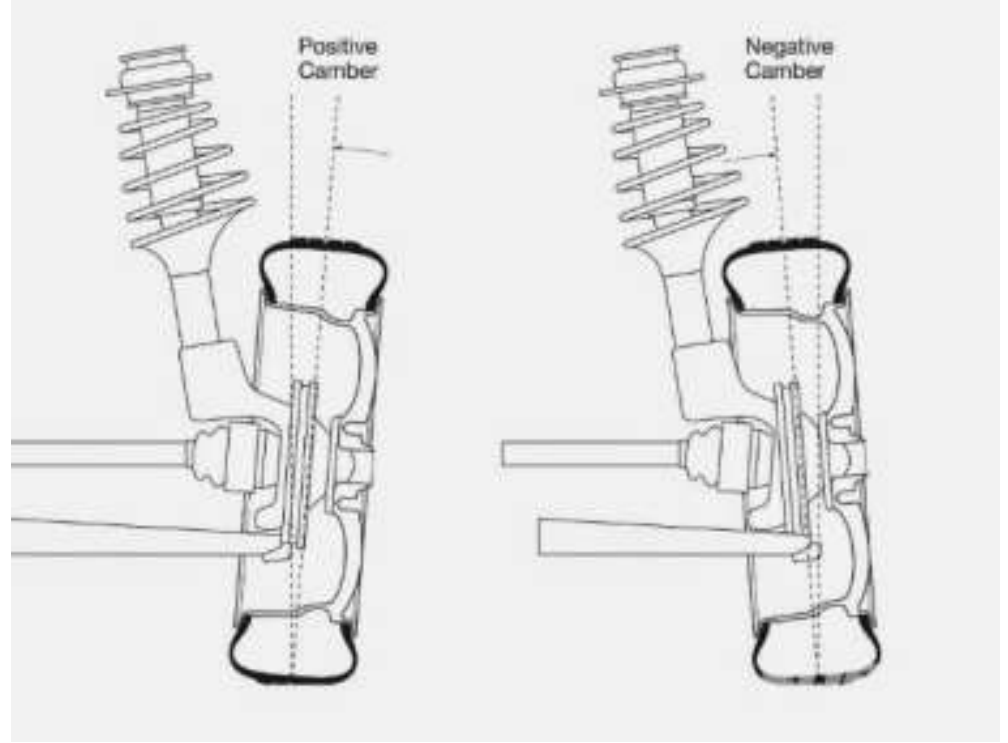

Figure 1: Types of Camber [3]

Positive camber may be described as the wheel's vertical axis falling outside (away from the vehicle) of the perpendicular axis formed between the road and the centre of the base of the wheel (the lowest point on contact with the road). Negative camber is simply the angle formed opposite to the positive one where the wheel's vertical axis falls inside of the perpendicular axis (toward the vehicle itself).

Effects of camber angle, for both positive and negative instances, can have dramatic effects on handling and grip. For example, a negative camber can have poor road surface contact for straight-line motion but may increase the contact between the surfaces of the road when cornering. Regardless, as the contact area negatively deviates from the maximum surface area allowed by the tire, the wear increases on said contact area. Ultimately too large of a camber angle can increase the wear per unit area for a small contact area of the wheel and may result in tire blowout.

A slightly negative camber is usually kept by most car manufacturers as it compensates for body roll effects which forces a positive camber during cornering [2]. The exact magnitude varies by manufacturer but can be appropriated to having a typical value of $-0.25^{\circ}$ according to Intrax Racing to $-0.5^{\circ}$ as reported by Seegert [4] since this represents a magnitude neither too large to reduce surface contact during straight line motion nor too small to compensate for typical cornering. Seegert [4] also reports that for some configurations as derived from the road conditions manufacturers target, that is the intended region the vehicles will be used within can result in the rear suspension being configured quite differently from the front suspensions, provided they are independent-type suspensions being used.

Caster, sometimes known as Castor, is the angle of the axis formed through the turning axis of the wheel, usually via the centreline of the McPherson Strut or the upper and lower ball joints, relative to the normal to the road's surface. Positive castor occurs when the axis line (shown in red in Fig. 2 below) leans toward the rear or away from the front of the vehicle. Conversely, negative castor is simply where the axis lineleans 
toward the front or away from the rear of the vehicle. It should be noted if not obvious that castor typically applies to the front wheels as the rear wheels do not exhibit a steering axis. Additionally, many vehicles lack a tunable castor and may be fixed.
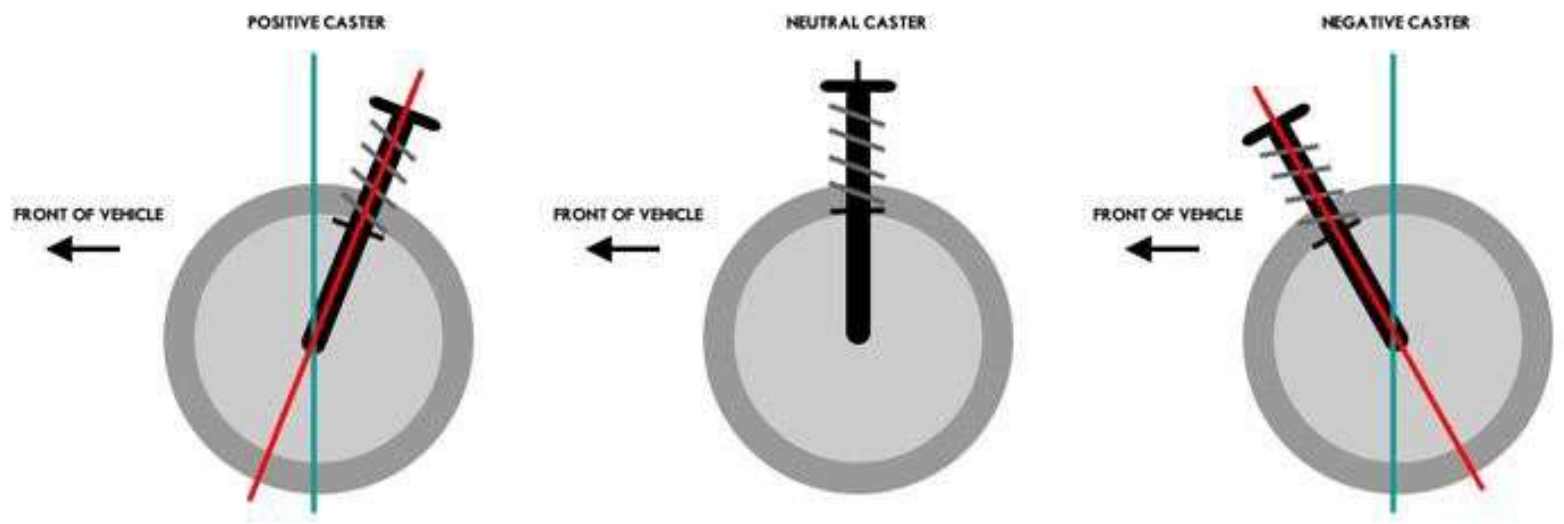

Figure 2: Types of Caster/Castor [5]

Castor is not usually considered to be "misaligned" like its sibling parameters. It may be adjusted to alter the handling of the vehicle and the manoeuvrability possible. For example, a positive castor can contribute a self-centring force allowing for straight-line movement without the need for holding or readjusting the steering wheel. The castor may be viewed therefore as the directional stability angle which corrects or alters the manoeuvrability vectors possible during cornering. Typically, castor is aligned at around $+7^{0} \pm 1$. [4]

Toe is the final category in the alignment criteria, though far from the ultimate parameter within the process of alignment. When undertaking a bird's-eye-view at the vehicle, an imaginary line superimposed about the wheel's riding direction through the longitudinal axis.

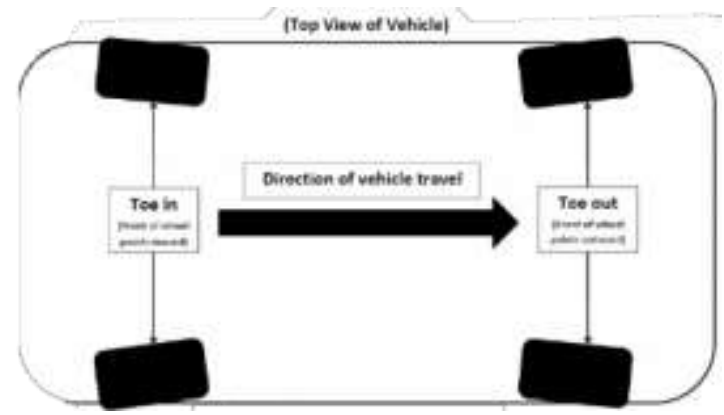

Figure 3: Overhead view of Toe Angles [6]

Seegert [4] claims that toe is typically regarded as the most common reason for accelerated tire wear and unstable handling. It is described as a V-shaped or Inverted-V (sometimes called A-shaped) when viewed from above or below [7]. This is called Toe-Out and Toe-In respectively.

To minimize tire wear, a zero-angle toe is recommended by Intrax Racing [2], that is the wheels are all parallel to each other. However, depending on the configuration of the car, that is to say: if the car possesses a front-wheel drive configuration or a rear-wheel drive configuration, the car may understeer or over/ steer. To compensate for this, a toe-out configuration for the front wheel-drive setup will introduce an "oversteer" thus effectively giving better cornering. Conversely, a toe-in can reduce oversteer already introduced in the rearwheel drive configuration and subsequently normalize the cornering angle. A key point to consider here is that toe is typically only applied to independent suspensions and as such in the presence of a rear- 
independent suspension, as in the case of sports-configured vehicles, toe begins to play a crucial role in the rear wheels.

Vehicles vary across the board with respect to their suspension configurations. Many light-trucks and cars may utilize a front-independent wheel suspension system with the rear semi-dependent or dependent suspension system, such as the solid axle system. In other cases, it may occur that all four wheels are supported via independent suspension systems. As such, since a sensor will be required to be mounted, it is important to evaluate these configurations.

Considering the differences between two wheel and four-wheel alignment firstly, it is necessary to state that four-wheel alignment is always the best practice. For the average driver, two-wheel alignment is always enough. Additionally, four-wheel alignment may not always be applicable depending on the rear wheel suspension system in place. For a fixed, dependent or semi-dependent rear suspension, the adjustments are usually non-existent or too difficult to perform. Should the rear axle possess a diffuse or offset positioning, that is it veers toward a side, it may result in a steering drift toward that side. As such, the typical solution lies in redirecting via repositioning the rear axle to compensate for the misalignment.

\section{Design Process}

The design involved breaking the problem into steps, then reviewing what possible solution methods could be implemented.

\subsection{Conceptual Design}

A functional analysis was used essentially as a means of representing an algorithmic overview for the data aspect, from collection to representation.

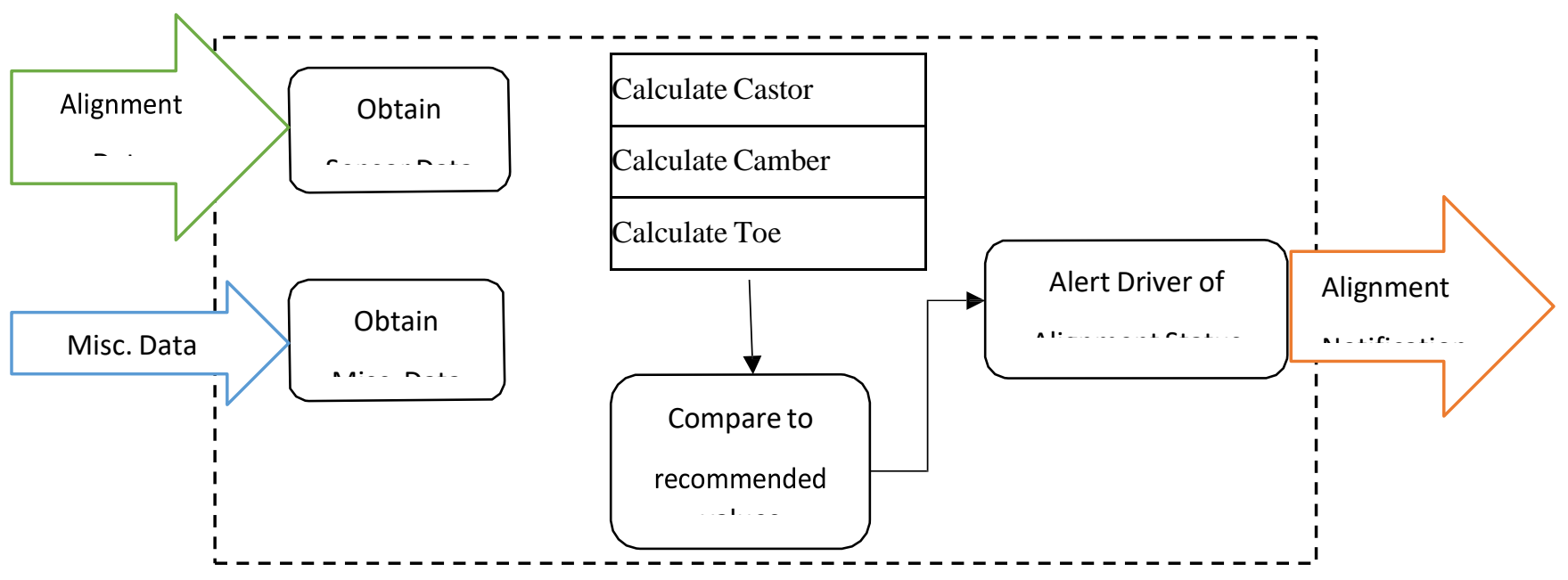

Figure 4: Simplified process for determining Alignment 
Table 1: A Morphological Chart of possible parametric configurations

\section{Energy/Data Method Sensor Method Suspension Type Alignment Mounting Method}

\begin{tabular}{|c|c|c|c|c|}
\hline \multicolumn{2}{|c|}{ Wired } & \multicolumn{3}{c|}{ Wireless } \\
\hline Electric & Magnetic & Acoustic & Optical & MEMS \\
\hline McPherson Strut & \multicolumn{2}{|c|}{ Double Wishbone } & \multicolumn{2}{c|}{ Multi-Link } \\
\hline \multicolumn{2}{|c|}{ 2-Wheel Alignment } & \multicolumn{3}{|c|}{ 4-Wheel Alignment } \\
\hline Mounting Bracket & Fixed & Mounting Arm & Adhesive \\
\hline
\end{tabular}

From the morphological chart as shown in Table.1 above, a list of alternatives was conceived. While this list can include every exhaustive combination from the table, only a subset may be relevant to the scope and limits of this study. As such, the shortlisted candidates are:

- Wired-Acoustic - McPherson Strut - 4-Wheel Alignment-Mounting Arm

- Wireless - Optical-McPherson Strut - Front Wheel Alignment-Mounting Bracket

- Wired-MEMS - Double Wishbone - Front Wheel Alignment-Fixed

The housing material is excluded from the selection at this stage as this involves a further iterative process. For the sake of simplification, all designs will use the Double Wishbone Suspension even if the alternative specifies otherwise.

\subsection{Alternative Designs}

\subsubsection{Design A: Wired-Acoustic-Arm}

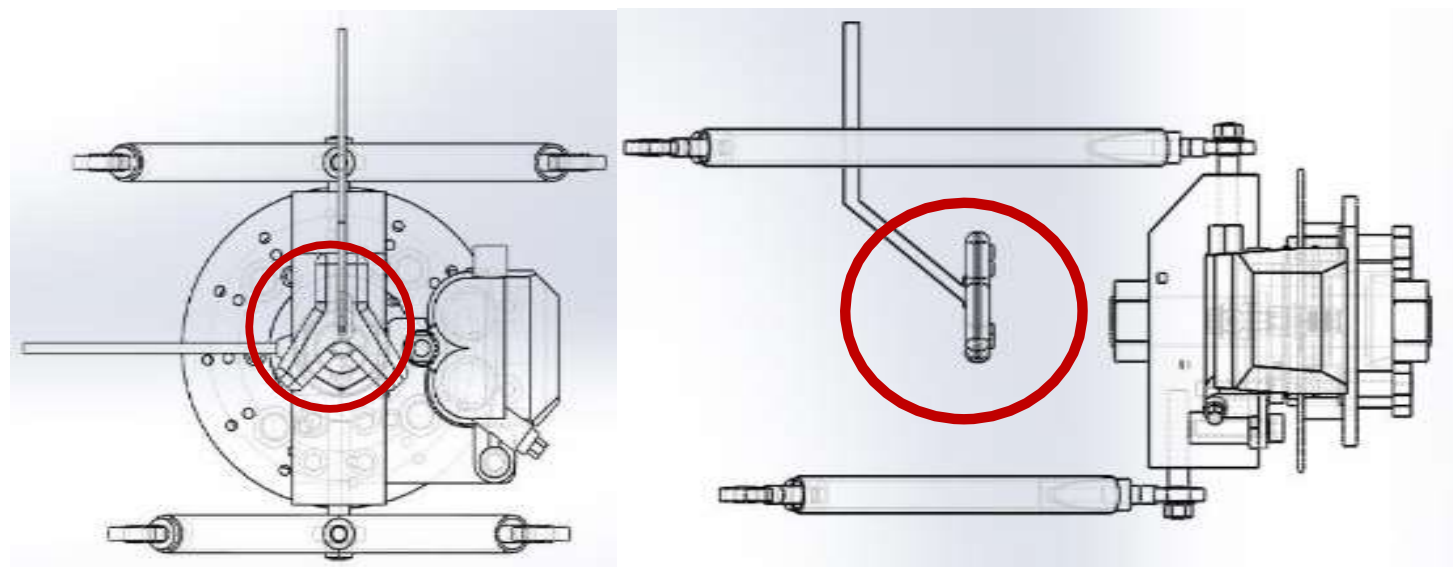

Figure 5: CAD drawings showing possible placement of the acoustic sensor module

Method:

1) Detect Start-Up

2) Test Noise Levels

3) Compare noise to known dataset. Is noise too high? YES? Set sensitivity. NO? Proceed to 4.

4) Measure distance from each of the acoustic sensors to varying positions on each wheel hub. 
5) Determine angle relative to referential plane.

6) Compare angles to recommended values. Are angles within bounds? NO? Alert Driver. YES? Exit.

\subsubsection{Design B: Wired-Optical-Bracket}
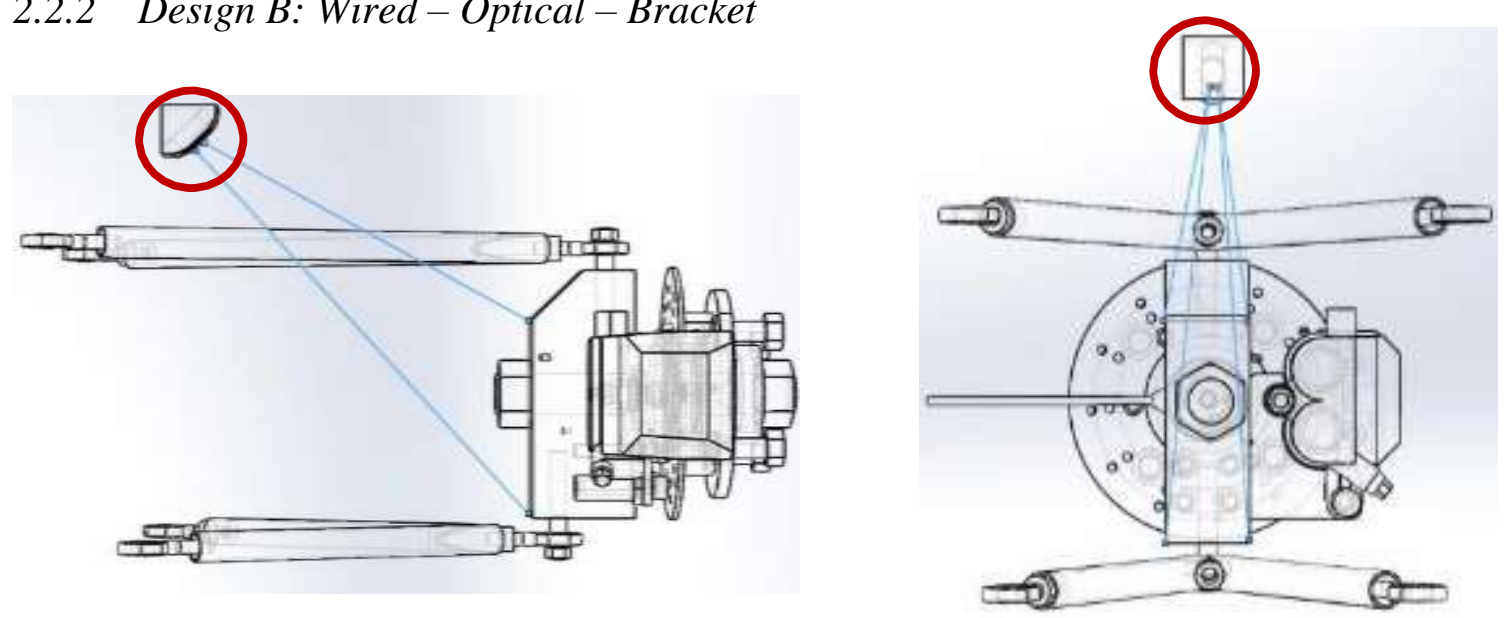

Figure 6: CAD drawings showing possible placement of the optical sensor module

Method:

1) Detect Start-Up

2) Calibrate with Optical Window Shutter Closed

3) Open Shutter Door.

4) Measure distance from floor. Adjust for even wheel plane if necessary.

5) Check wheel hub assembly edge profile.

6) Compare to boundary conditions. Is edge date out of bounds? YES? Alert driver. NO? Exit.

\subsubsection{Design C: Wired-MEMS - Fixed}
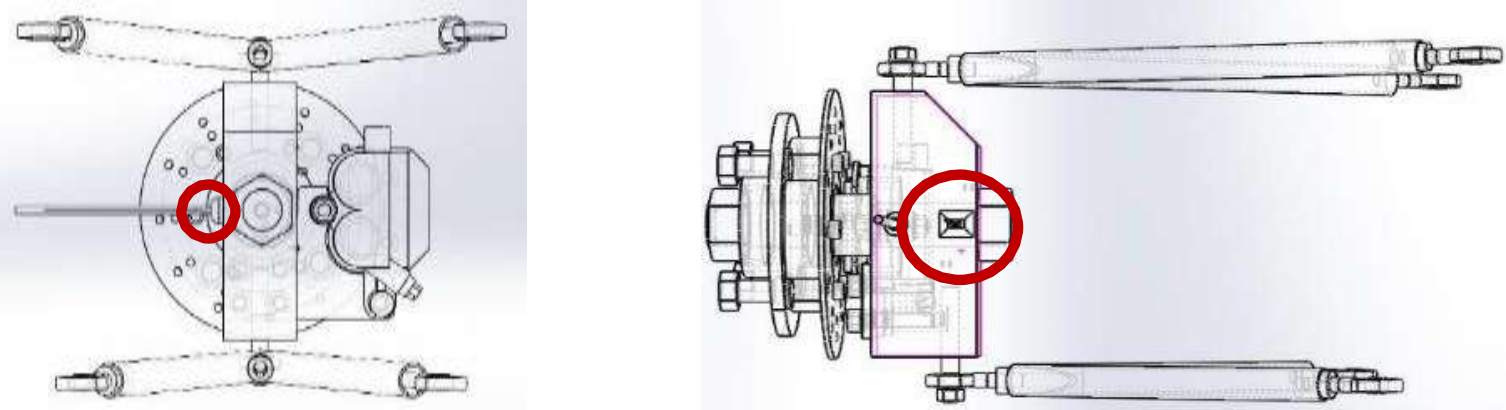

Figure 6: CAD drawings showing possible placement of MEMs module

Method:

1) Detect Start-up

2) Read orientation data and temperature data from calibration sensor (located at a midplane referential position on the chassis), as well as from the sensors on each wheel hub. 
3) Adjust for errors due to thermal deviation.

4) Compare wheel hub sensors to recommended values. Are parameters out of bounds? NO? Exit. YES? Alert Driver.

\subsection{Design Evaluation}

Table 2: Pugh Concept Screening Chart

$$
\begin{aligned}
& \text { Selection Criteria } \\
& \text { Ease of Use } \\
& \text { Ease of Integration } \\
& \text { Manufacturing Ease } \\
& \text { Cost } \\
& \text { Accuracy of Results }
\end{aligned}
$$

Concept Variants

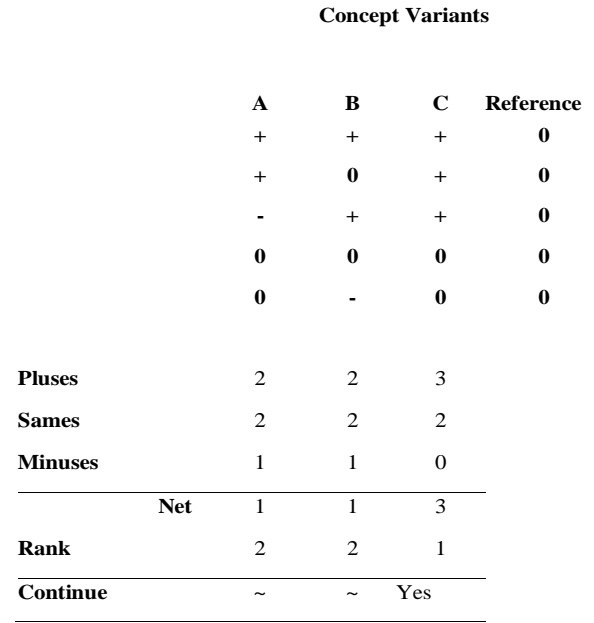

Note: $\sim$ indicates secondary alternatives to the leader.

Since the study required one candidate to proceed with, Design C was selected after carrying out a Pugh Screening Test as shown in Table. 2 above.

\section{Results}

The results obtained were benchmarked against a Hunter ProAlign Laser Alignment System at a professional Alignment Centre. They were as follows:

Table 3: Camber results displayed in degrees.

\begin{tabular}{|l|l|l|l|l|l|l|l|l|c|}
\hline Reference & $\begin{array}{l}\text { Left } \\
\text { Reading }\end{array}$ & $\begin{array}{l}\text { Left } \\
\text { Camber }\end{array}$ & $\begin{array}{l}\text { Right } \\
\text { Reading }\end{array}$ & $\begin{array}{l}\text { Right } \\
\text { Camber }\end{array}$ & $\begin{array}{l}\text { Actual } \\
\text { Left } \\
\text { Camber }\end{array}$ & $\begin{array}{l}\text { Actual } \\
\text { Right } \\
\text { Camber }\end{array}$ & $\begin{array}{l}\text { Left } \\
\text { Error }\end{array}$ & $\begin{array}{l}\text { Right } \\
\text { Error }\end{array}$ & $\begin{array}{l}\text { Intended } \\
\text { Camber }\end{array}$ \\
\hline 232.05 & 232.06 & 0.01 & 232.03 & 0.02 & -0.10 & 0.00 & 0.11 & 0.02 & $\mathbf{0}$ \\
\hline 232.07 & 230.93 & -1.14 & 233.01 & -0.94 & -1.20 & -1.30 & 0.06 & 0.36 & $\mathbf{- 1}$ \\
\hline 232.02 & 229.96 & -2.06 & 234.07 & -2.05 & -2.00 & -2.20 & -0.06 & 0.15 & $\mathbf{- 2}$ \\
\hline 232.03 & 229.01 & -3.03 & 235.05 & -3.02 & -2.90 & -3.10 & -0.13 & 0.08 & $\mathbf{- 3}$ \\
\hline 232.07 & 233.08 & 1.01 & 230.98 & 1.09 & 1.00 & 0.90 & 0.01 & 0.19 & $\mathbf{1}$ \\
\hline 232.08 & 234.01 & 1.94 & 230.01 & 2.07 & 2.00 & 2.00 & -0.06 & 0.07 & $\mathbf{2}$ \\
\hline 232.07 & 235.41 & 3.34 & 228.98 & 3.09 & 3.20 & 3.10 & 0.14 & -0.01 & $\mathbf{3}$ \\
\hline
\end{tabular}

Table. 3 was summarized from a larger data set comprising of 10 data points per Camber variation, taken at 1 second intervals. There were 7 variations in total for both the left and right sensor, with an intended range of - 
$3 \leq \Theta$ camber $\leq 3$. However, since obtaining these intended angles were done by manually adjusting components in the suspension, in the interest of time (as the alignment service was being paid for on a per session basis) approximations were made to these with their 'true' values as recorded by the Alignment machine. Thus, this machine served as a benchmark and the data obtained from the machine is listed as "Actual Camber." Raw data obtained from the IMU sensor was listed as Left Reading and Right Reading for both the left and right wheel respectively, especially when facing the front of the car. As these values

were absolute according to the sensors, they conveyed no meaning except for calculating the relative readings. Here the Left Camber and Right Camber were calculated using a simple formula.

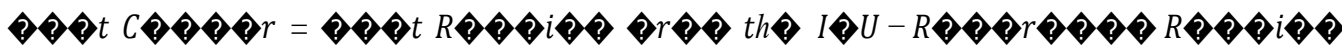

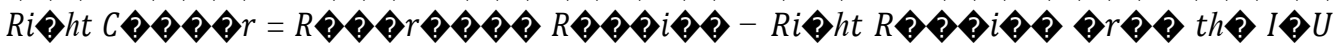

The variation in order of subtraction occurring in either of the equations was due to an established convention set at the beginning of the study. As the viewing plane was that of an onlooker facing the front of the vehicle, if the top of the wheel extended toward the vehicle (and subsequently the base extended away), then this system would possess a negative camber (refer to Figure 1). Thus, when the wheels were given a known set of camber values: zeroed, negative and positive, their responses were measured, recorded and compared. The accuracies of the prototype IMU network compared to the benchmark are seen in Fig. 7 and Fig. 8 below, for the Left and Right Camber Angles (in degrees) respectively.

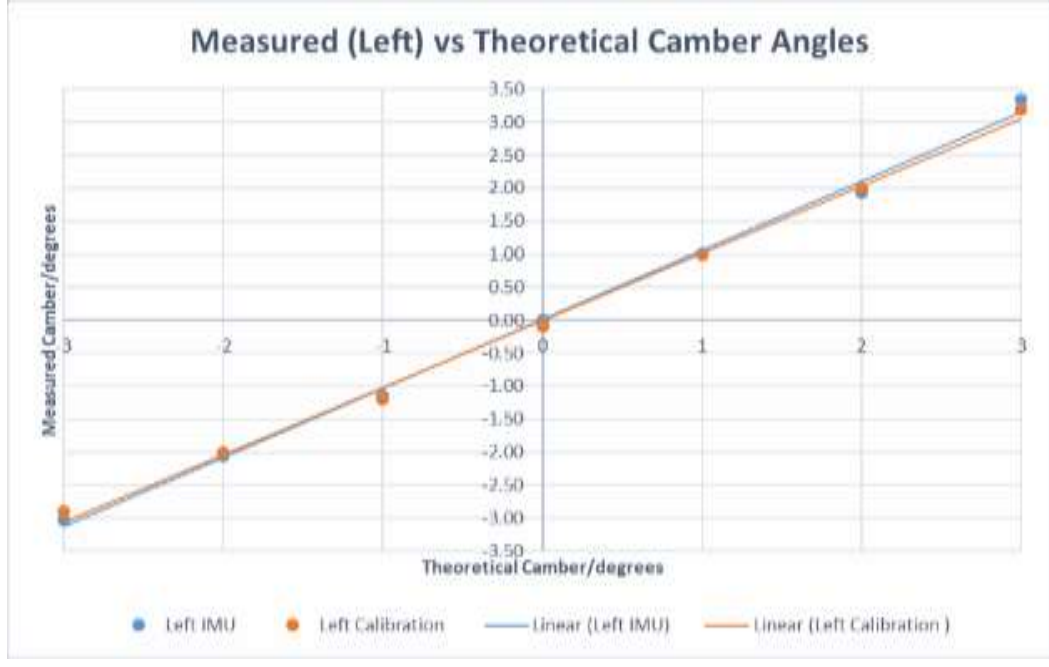

Figure 7: Graph comparing measured vs benchmark camber data for the front-left wheel 
The International Conference on Emerging Trends in Engineering and Technology (IConETech-2020)

Faculty of Engineering, The UWI, St. Augustine | June $1^{\text {st }}-5^{\text {th }}, 2020$

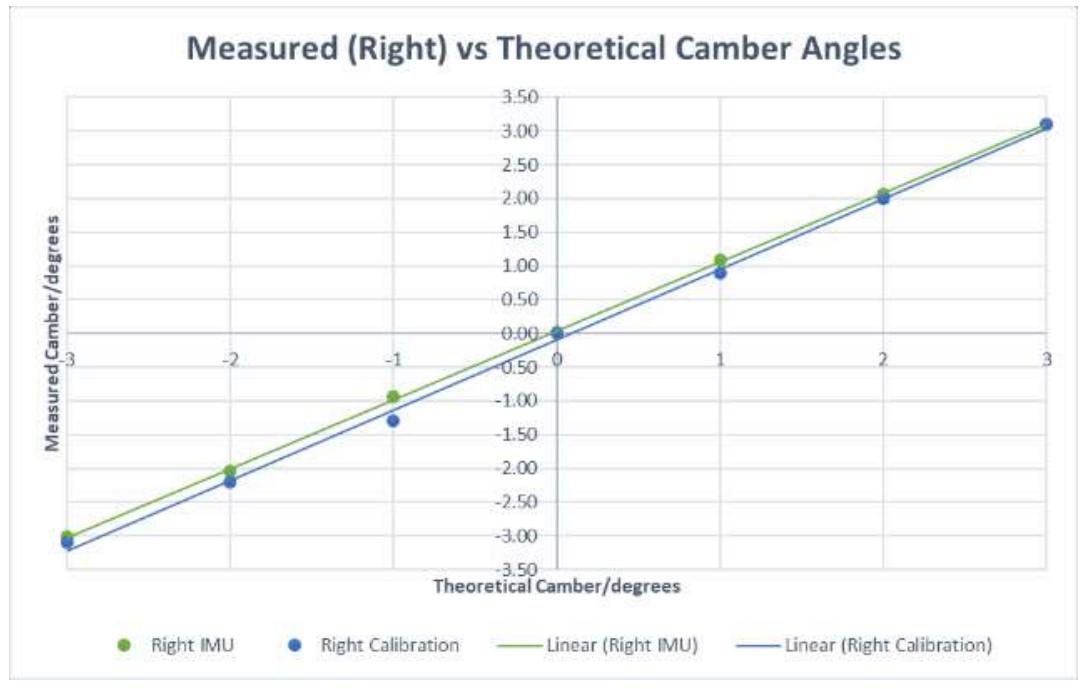

Figure 8: Graph comparing measured vs benchmark camber data for the front-right wheel 
Table 4: Measured Toe values for the IMU vs Benchmark in degrees

\begin{tabular}{|l|l|l|l|l|l|l|l|l|l|l|l|}
\hline Ref & $\begin{array}{l}\text { Left } \\
\text { Raw }\end{array}$ & $\begin{array}{l}\text { Right } \\
\text { Raw }\end{array}$ & $\begin{array}{l}\text { Left } \\
\text { IMU }\end{array}$ & $\begin{array}{l}\text { Right } \\
\text { IMU }\end{array}$ & Prefix & $\begin{array}{l}\text { Toe } \\
\text { IMU) }\end{array}$ & $\begin{array}{l}\text { Left } \\
\text { Cal }\end{array}$ & $\begin{array}{l}\text { Right } \\
\text { Cal }\end{array}$ & Prefix & $\begin{array}{l}\text { Toe } \\
\text { (CAL) }\end{array}$ & Alert \\
\hline 91.01 & 90.95 & 91.11 & -0.06 & -0.10 & $\mathbf{0}$ & OUT & 0.0 & 0.0 & $\mathbf{0}$ & Neutral & No \\
\hline 90.98 & 93.04 & 88.44 & 2.06 & 2.54 & $\mathbf{0}$ & IN & 1.5 & 2.0 & $\mathbf{0}$ & IN & No \\
\hline 90.99 & 87.54 & 95.18 & -3.45 & -4.19 & $\mathbf{0}$ & OUT & -4.0 & -4.0 & $\mathbf{0}$ & OUT & No \\
\hline 91.02 & 87.79 & 91.46 & -3.23 & -0.44 & $\mathbf{E}$ & OUT & -3.0 & 0.0 & $\mathbf{E}$ & OUT & Amber \\
\hline 91.00 & 94.01 & 91.04 & 3.01 & -0.04 & $\mathbf{E}$ & IN & 3.0 & 0.0 & $\mathbf{E}$ & IN & Amber \\
\hline 91.05 & 90.61 & 90.31 & -0.44 & 0.74 & $\mathbf{0}$ & Neutral & -0.5 & 0.5 & $\mathbf{0}$ & Neutral & No \\
\hline 91.04 & 92.31 & 88.02 & 1.27 & 3.02 & $\mathbf{E}$ & IN & 1.5 & 3.0 & $\mathbf{E}$ & IN & Amber \\
\hline 91.03 & 99.41 & 84.01 & 8.38 & 7.02 & $\mathbf{X}$ & IN & 8.0 & 7.0 & $\mathbf{X}$ & IN & Red \\
\hline 90.97 & 83.35 & 98.71 & -7.62 & -7.74 & $\mathbf{X}$ & OUT & -7.5 & -7.5 & $\mathbf{X}$ & OUT & Red \\
\hline 91.02 & 90.17 & 83.82 & -0.85 & 7.20 & $\mathbf{X}$ & OUT & -1.0 & 7.0 & $\mathbf{X}$ & IN & Red \\
\hline
\end{tabular}

Analysis of the Toe Data set was, like the data from the Camber Summary, found to be reasonably accurate to that of the benchmark. Again, the same equations for determining the relative angles of the Left and Right Wheels were utilized with similar sign conventions, albeit using a different plane. However, as there were many more variations present this time for qualifying not just Toe-position but the "magnitude" of toe as well, it required creating a standard to work with.

Table 5: Simplified standard used to represent data in Table 4.

Toe Property Angle

\begin{tabular}{l|l}
\hline IN & $>+1$ \\
OUT & $<-1$ \\
Neutral & Approximately 0, i.e. $-1 \rightarrow+1$
\end{tabular}

Table 6: Modifier parameters used in Table 4.

Modifier

Parameter

\begin{tabular}{l|l}
\hline$O$ & Both wheels ideally Toe'd. \\
$E$ & $1-$ wheel Neutral, 1-wheel Toe'd, but still in acceptable range. \\
$X$ & Either or Both Wheels not in acceptable range. Increased wear.
\end{tabular}

\section{Conclusion}

Utilizing the IMU sensor in parallel for this study, it was shown that a device could be developed for use with vehicles and integrated in a manner like that already used for ABS Sensors on a wheel hub. Comparing the data obtained, it was found the device could operate within an acceptable margin of error to produce high quality orientation data on par with what could be obtained from a professional grade tool (the Hunter Alignment System). Since wheel alignment is something very few think about but can directly increase wear on a vehicle, giving a feedback system to the driver in the same manner as a fuel level indicator can prove to be an invaluable asset. 


\section{References}

[1] Knowles, D. (2002). Automotive Suspension and Steering Systems (3rd ed.). Cengage Learning. [2] Intrax Racing. (2015). Camber, Caster, Toe-in.out explained. Retrieved October 2, 2015, from Intrax Racing: http://en.intraxracing.nl/techniek/camber,-caster,-toe-intoe-out/

[3] Hau, J. (2014, May 5). Wheel Camber. Retrieved October 12, 2015, from Jinhau - Wordpress: https://jinhau.files.wordpress.com/2014/05/581e6-wheelcamber.jpg

[4] Seegart, R. (2008, April 16). TJ and LJ Wheel Alignment from Wandering Trail:

http://www.wanderingtrail.com/Mechanical/TJLJ/Diagnostic_Adjust_Repair/Steering_Alignment /Alignment.htm

[5] Viking Speed Shop. (2015, January). Caster. Retrieved October 12, 2015, from Viking Speed Shop: http://www.vikingspeedshop.com/wp-content/uploads/caster.jpg

[6] Autocraze Austrailia. (2015). Toe-Adjustment. Retrieved October 3, 2015, from Autocraze: http://www.autocraze.com.au/wp-content/uploads/2015/05/toe-adjustment.png

[7] Reimpell, J., Stoll, H., \& Betzler, J. (2001). The Automotive Chassis: Engineering Principles (2nd ed.). (A. Limited, Trans.) Woburn, MA: Society of Automotive Engineers.

[8] Genta, G., \& Morello, L. (2009). The Automotive Chassis (Vol. 1). (F. F. Ling, Ed.) Torino, Italy: Springer.

[9] Genta, G., \& Morello, L. (2009). The Automotive Chassis (Vol. 2). (F. F. Ling, Ed.) Torino, Italy: Springer.

[10] Graham Hathaway Racing. (2015, January). image5. Retrieved October 12, 2015, from Graham Hathaway Racing: http://www.grahamhathawayracing.com/assets/images/image5.jpg

[11] Society of Automotive Engineers. (2013, February 20). Vehicle Alignment Basics. Retrieved October 11, 2015, from SAE International: http://www.sae.org/search/?qt=camber\&sort=relevance\&sortdir $=$ desc $\&$ display $=$ list $\&$ content - type $=\% 28 \% 22$ ARTICLE $\% 22 \% 29$ 